\title{
Benign paroxysmal positional vertigo: Diagnostic criteria
}

\author{
Consensus document of the Committee for the Classification of Vestibular Disorders of the Bárany \\ Society
}

\author{
Michael von Brevern ${ }^{\mathrm{a}, *}$, Pierre Bertholon ${ }^{\mathrm{b}}$, Thomas Brandt ${ }^{\mathrm{c}}$, Terry Fife ${ }^{\mathrm{d}}$, Takao Imai ${ }^{\mathrm{e}}$, \\ Daniele Nuti ${ }^{\mathrm{f}}$ and David Newman-Toker ${ }^{\mathrm{g}}$ \\ ${ }^{a}$ Department of Neurology, Park-Klinik Weissensee, Berlin, Germany \\ ${ }^{\mathrm{b}}$ Department of Otolaryngology - Head and Neck Surgery, Bellvue Hospital, Saint-Etienne, France \\ ${ }^{\mathrm{c}}$ Institute of Clinical Neuroscience, Ludwig-Maximilian University, Munich, Germany \\ ${ }^{\mathrm{d}}$ Barrow Neurological Institute, University of Arizona College of Medicine, Phoenix, AZ, USA

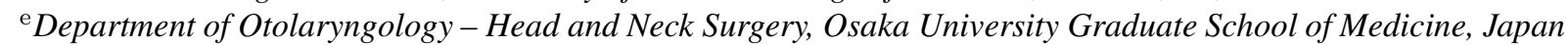 \\ ${ }^{\mathrm{f}}$ Department of Otolaryngology - Head and Neck Surgery, University of Siena, Italy \\ ${ }^{g}$ Department of Neurology, Johns Hopkins University School of Medicine, Baltimore, MA, USA
}

Received 2 May 2014

Accepted 3 June 2015

\begin{abstract}
This article presents operational diagnostic criteria for benign paroxysmal positional vertigo (BPPV), formulated by the Committee for Classification of Vestibular Disorders of the Bárány Society. The classification reflects current knowledge of clinical aspects and pathomechanisms of BPPV and includes both established and emerging syndromes of BPPV. It is anticipated that growing understanding of the disease will lead to further development of this classification.
\end{abstract}

Keywords: Vertigo, positional, nystagmus, diagnosis, criteria

\section{Introduction}

The following diagnostic criteria for benign paroxysmal positional vertigo (BPPV) are part of the International Classification of Vestibular Disorders (ICVD) - an endeavour for classification of vestibular disorders steered by the Committee for Classification of Vestibular Disorders of the Bárány Society. As a first step and prerequisite for the classification of vestibular disorders, the Classification Committee of the Bárány Society published a consensus on the definition of vestibu-

*Corresponding author: Michael von Brevern, Department of Neurology, Park-Klinik Weissensee, Schönstrasse 80, 13086 Berlin, Germany. Tel.: +49 30 96283700; Fax.: +49 30 96283705; E-mail: von.brevern@park-klinik.com. lar symptoms [13]. Individual subcommittees including otolaryngologists and neurologists from at least three continents have been tasked with defining consensus diagnostic criteria for specific vestibular disorders.

We have taken additional steps to ensure the broadest possible consensus for the criteria. The classification has been presented and discussed at two meetings of the Bárány Society in 2010 and 2012. Furthermore, a draft of the classification was presented to five other medical societies engaged in neurotology (American Academy of Otolaryngology - Head and Neck Surgery, American Neurotology Society, American Otological Society, European Academy of Otology and Neuro-Otology, and Japan Society of Equilibrium Research). The members of the Bárány-Society were invited to comment on the last draft of this clas- 
sification before publication by means of an online internal review system. Comments from Bárány Society members and from the consulted medical societies were discussed and, where appropriate, incorporated by the subcommittee before the final version of this classification was approved. Finally, we have taken steps to ensure that our document is harmonious with current published therapeutic guidelines from the American Academy of Neurology [31] and the American Academy of Otolaryngology - Head and Neck Surgery [11].

The format of this classification is modelled on the International Classification of Headache Disorders. It includes both established syndromes (Sections 2.1 to 2.4) and emerging and controversial syndromes (Sections 3.1 to 3.4 ). The operational criteria are supported by notes and comments.

\subsection{Terminology}

Because symptoms are triggered by the act of moving the head to a new position, rather than by maintaining the head in a particular posture or position, some authors have advocated for the use of the term "positioning" vertigo rather than "positional" vertigo for BPPV. A similar issue arises in distinguishing "positioning" nystagmus from "positional" nystagmus on the basis of whether the finding is transient after head positioning. While these distinctions make mechanistic sense and may be of diagnostic value, it was the group's judgment that current usage of the term "positional" in BPPV or to describe its associated nystagmus is too ubiquitous to change clinical practice. Thus, the term "positional" is preserved in the definitions below and "positioning" is not used.

\subsection{Epidemiology}

BPPV is the most frequent vestibular disorder: its cumulative incidence in the general population amounts during lifetime to $10 \%$ [79]. The time course of BPPV is characterised by spontaneous remissions that occur typically after days to weeks [41] and recurrences that occur in about $50 \%$ of patients [61]. Although BPPV is usually self-limiting, it inflicts a considerable personal and socio-economic burden [55 79].

\subsection{Pathophysiology}

According to a widely accepted theory BPPV is usually caused by otoconia that are dislodged from the otolith macula beds and are trapped in a semicircular canal. Gravity causes them to move after changes of the head position in the plane of the affected canal. The resulting inappropriate endolymph flow deflects the cupula and thus modulates the activity of the vestibular afferents of the affected canal, causing attacks of positional vertigo and nystagmus (canalolithiasis) [15]. Less common, BPPV can be attributed to otoconia that are attached to the cupula of a semicircular canal and render it sensitive to gravity (cupulolithiasis) [5|51/73]. The development of pathophysiological concepts of BPPV that aimed to understand the clinical features of the disease as a mechanical irritation of a semicircular canal has been an intellectual challenge [15 50]. Schuknecht coined the term cupulolithiasis on the basis of histological studies of the temporal bones from patients with BPPV [67]. Incidentally, he also has provided a documentation of canalolithiasis, although he did not recognize the pathophysiological meaning of this finding [67].

The critical role of the receptors of the semicircular canals is supported by the direction of positional nystagmus in BPPV reflecting the known excitatory and inhibitory connections of the canal receptors with specific extraocular eye muscles [2]. The hypothesis of canalolithiasis and cupulolithiasis is supported by in-vitro and in-vivo animal models [6371], by mathematical models [33 4065] and by the efficacy of specific canal clearing procedures [31|32|54] and plugging of the affected semicircular canal [10]. Furthermore, particulate matter within the posterior canal has been observed intraoperatively in patients with BPPV [64:80]. This debris has been examined by scanning electron microscopy and appeared morphologically consistent with degenerated otoconia [80]. Of note, particulate matter has also been found in the posterior semicircular canal of subjects without a history of BPPV [49 59]. This finding is not contradictory to the pathophysiology of BPPV as discussed above as physio-mathematical models have shown that the prerequisite for BPPV is a certain amount of otoconia within the affected semicircular canal reaching a "critical mass" and that an agglomeration of these particles promotes the hydrodynamic effect of otoconia moving in the canal [3340].

There are open questions that need to be addressed in the future. It has been postulated that loose otoconia within the short arm of the semicircular canals (on the utricular side of the cupula) may also cause positional vertigo [17/18]. Furthermore, canalolithiasis and cupulolithiasis are not mutually exclusive and may co- 
exist simultaneously in the same semicircular canal but such a combination has not been documented with eye movement recording so far.

There are clinical findings that are possibly associated with BPPV but not yet sufficiently understood. Spontaneous nystagmus not provoked by positioning is not a core feature of BPPV, although "pseudospontaneous" nystagmus is typical of horizontal canal BPPV (see comment to 1.3 further below). Rarely, however, spontaneous nystagmus beating in a direction that corresponds to the stimulation of a single semicircular canal irrespective of head position has been observed in patients that otherwise fulfilled diagnostic criteria for canalolithiasis or cupulolithiasis. This finding is usually brief, rarely lasting longer than a few minutes and has been described as a complication of positional therapy and after shaking the head in the plane of the canal affected by canalolithiasis [2876] and may possibly also occur spontaneously [58]. The pathophysiology is unclear. A "canalith jam" implying a locking of the cupula in a fixed position by canalith debris, either directly jamming the cupula or indirectly blocking endolymph flow in the canal has been postulated [28 58 76].

Finally, patients with persistent geotropic directionchanging positional nystagmus in the supine lateral head positions in the absence of central neurologic signs and with normal results in cerebral imaging have been described [3744]. This type of nystagmus is neither compatible with mobile otoconia in a semicircular canal nor with dense otoconia attached to a cupula. However, there seems to be an association between persistent geotropic direction-changing positional nystagmus and canalolithiasis and cupulolithiasis, as some of these patients have developed classical variants of BPPV at follow-up [37 44]. It has been suggested that this phenomenon may be due to changes of the density of the cupula or the endolymph [37/44], but this remains speculative.

\subsection{Diagnosis}

The complete diagnosis includes the specification of the affected semicircular canal(s) and the pathophysiology (canalolithiasis or cupulolithiasis). The definite diagnosis of BPPV requires diagnostic positional maneuvers that lead to the observation of a canal-specific positional nystagmus. Clinical features essential for the diagnosis are the latency, direction, time course, and duration of positional nystagmus. Usually, further vestibular and auditory testing is indicated only when a pre-existing disorder of the inner ear (e.g. vestibular neuritis, Menière's disease) is suspected. Similarly, brain or ear imaging is not required in typical cases of BPPV [11].

Positional testing involves the provocation of vertigo and nystagmus, and different maneuvers test different semicircular canals. A canal-specific response is diagnosed when a rotation of the head in the plane of a semicircular canal evokes positional nystagmus of maximal intensity (in terms of slow phase velocity). As a rule (and in contrast to central positional nystagmus), positional nystagmus in BPPV always beats in the plane of the affected canal and in the expected direction for canal excitation or inhibition [19].

Basically, the head is brought first into such a position that the affected semicircular canal is spatially vertical and thus aligned with gravity. In a second step, the head is then rotated in the plane of the affected canal. In the Dix-Hallpike maneuver, the head of the sitting patient is turned $45^{\circ}$ toward the side to be tested and then laid back quickly into a head-hanging position [27]. In the side-lying maneuver (Semont diagnostic maneuver) the sitting patient is tilted quickly to the side to be tested with the head turned $45^{\circ}$ to the opposite side [24]. It is noted that both maneuvers not only test for lithiasis of the posterior canal of one side but also for the anterior canal of both sides [26 16]. For testing of the horizontal canal, the supine roll test is used: the head of the patient in the supine position is elevated about $30^{\circ}$ and then turned quickly to either side. It is essential to perform positional maneuvers for both the vertical and the horizontal semicircular canals in every patient with positional vertigo as multiple canals may be affected [8[60].

For observation of positional nystagmus, Frenzel goggles or video-oculography can be helpful, particularly when the nystagmus is weak or momentary. Recording of eye movements may enhance one's ability to observe and to classify nystagmus in canalolithiasis of the anterior canal (3.1), in cupulolithiasis of the posterior canal (3.2), in lithiasis of multiple canals (3.3.) and in possible BPPV (3.4). In most cases, however, nystagmus can be seen clinically without special equipment. The direction of nystagmus is essential to specify the affected canal. Indeed, the posterior canal is by far the most frequently affected canal (8090\%); next is the horizontal canal (5-30\%) [25]3873]. Involvement of the anterior canal is rare, accounting for only $1 \%$ to $2 \%$ of patients in large case series [3860 81] although some recent reports have suggested a considerably higher incidence [20 45]. Theo- 
retically, each of the three semicircular canals of the labyrinth can be affected by canalolithiasis or cupulolithiasis, resulting in six combinations (not counting multiple canal involvement) for each ear. Except for cupulolithiasis of the anterior canal, all these combinations have been documented by eye movement recordings and are included in this classification.

Therapeutic positional maneuvers are highly effective for treatment of BPPV, particularly when canalolithiasis is present [1131]. Thus, if positional nystagmus disappears immediately after positional therapy, this strongly supports the diagnosis of BPPV. Such a favourable response to treatment is not mandatory for the diagnosis, and patients refractory to treatment do occur. However, repeated lack of response to therapy should generally prompt consideration of alternative diagnoses that may mimic BPPV closely [11].

The differential diagnosis of BPPV includes central positional vertigo due to vestibular migraine [77] and structural brainstem and cerebellar lesions, typically in the vicinity of the fourth ventricle [14]. CNS disease can usually be excluded by a thorough neurological examination, but some cases may be challenging to diagnose [48]. Greater care should be taken in patients with dominantly horizontal or downbeat positional nystagmus forms, since these are most frequently reported in central mimics [9 46 48]. Cerebral imaging with MRI is usually only indicated when symptoms or signs of concurrent brainstem or cerebellar dysfunction are present, or when positional vertigo and nystagmus present with atypical features or fail to resolve with repeated therapeutic positional maneuvers [11].

\section{Diagnostic criteria for benign paroxysmal positional vertigo}

Previously used terms: benign paroxysmal positioning vertigo, benign positional vertigo, paroxysmal positional vertigo, vestibular lithiasis.

\subsection{Canalolithiasis of the posterior canal (pc-BPPV)}

A. Recurrent attacks ${ }^{1}$ of positional vertigo or positional dizziness ${ }^{2,3,4}$ provoked by lying down or turning over in the supine position ${ }^{5}$.

B. Duration of attacks $<1 \min ^{6}$.
C. Positional nystagmus ${ }^{7}$ elicited after a latency of one or few seconds ${ }^{8}$ by the Dix-Hallpike maneuver or side-lying maneuver (Semont diagnostic maneuver). The nystagmus is a combination of torsional nystagmus with the upper pole of the eyes beating toward the lower ear combined with vertical nystagmus ${ }^{9}$ beating upward (toward the forehead) typically lasting $<1$ minute $^{10,11,12,13}$.

D. Not attributable to another disorder ${ }^{14}$.

\section{Notes}

1. In addition to attacks of positional vertigo, patients may have prolonged mild unsteadiness [26], even after successful treatment of benign paroxysmal positional vertigo [66].

2. BPPV typically leads to positional vertigo but occasionally patients may complain of positional dizziness.

3. Other complaints during the attacks include external vertigo (the visual sense of environmental motion that often accompanies the internal vestibular sense) [13], unsteadiness, and vegetative symptoms such as nausea, sweating, and tachycardia.

4. Positional vertigo or dizziness must be distinguished from orthostatic symptoms present only on arising but not with other positional triggers [13].

5. Attacks may not only be provoked in bed but also by other head movements (e.g., tilting the head backward [i.e., chin upward] or forward).

6. Patients may overestimate the duration of single attacks and mild, residual symptoms after an attack occasionally last minutes or hours. Furthermore, attacks may be triggered repetitively, causing more extended symptoms. The duration of vertigo should not, however, generally exceed 1 minute; single attacks lasting consistently longer should be considered atypical and spark consideration of alternate or additional diagnoses.

7. If positional nystagmus disappears immediately after positional therapy, this further supports the diagnosis.

8. The latency between the completion of the diagnostic positional maneuver and the onset of positional nystagmus may be as long as 40 seconds in rare cases [3].

9. The torsional component of positional nystagmus is slightly more prominent in the lower eye, 
whereas the vertical component is slightly more prominent in the upper eye [39]. If fixation is not suppressed, the nystagmus may appear dominantly torsional, since fixation's ability to suppress torsional eye movements is limited relative to suppression for vertical movements. The direction of the patient's gaze may influence the appearance of positional nystagmus. If gaze is directed to the lower ear, nystagmus may appear predominately torsional; if directed to the upper ear, it may appear predominantly vertical [14]. Independent of orbital eye position, the eye movement in a head-referenced coordinate system remains fixed in the plane of the posterior semicircular canal.

10. Usually, the duration of positional nystagmus does not exceed 40 seconds before it damps spontaneously [3].

11. Typically, positional nystagmus increases rapidly in intensity and then declines more slowly (crescendo-decrescendo) [3].

12. Nystagmus of lower intensity with reversed direction may appear after the initial positional nystagmus has ceased [3].

13. After the patient returns to the upright position, positional nystagmus with reversed direction of lesser intensity and shorter duration often occurs. Furthermore, fatigability of nystagmus and vertigo with repetitive positional testing is a common finding.

14. History and physical and neurological examinations do not suggest another vestibular disorder or such a disorder is considered but ruled out by appropriate investigations or such disorder is present as a comorbid condition that can be clearly differentiated.

\section{Comments}

Canalolithiasis of the posterior canal is by far the most common variant of benign paroxysmal positional vertigo, accounting for $80-90 \%$ of cases [60]. The right labyrinth is affected slightly more often than the left [78].

The occurrence of a bilateral canalolithiasis of the posterior canal is not rare, but is more prevalent in posttraumatic cases than in idiopathic canalolithiasis [47]. Note that unilateral benign paroxysmal positional vertigo may mimic bilateral benign paroxysmal positional vertigo of the posterior canal if the positional maneu- ver is executed without appropriate alignment of the head [68].

The occurrence of canalolithiasis of the posterior canal without positional nystagmus is controversial but improvement of positional vertigo after a therapeutic positional maneuver in such patients with a typical history of benign paroxysmal positional vertigo has been reported [34/72]. These patients should be coded as 3.4. Possible benign paroxysmal positional vertigo. It is conceivable that the number of otoconia in the posterior canal is sufficient to evoke subjective symptoms but insufficient to stimulate the vestibulo-ocular reflex [40].

\subsection{Canalolithiasis of the horizontal canal (hc-BPPV)}

A. Recurrent attacks ${ }^{1}$ of positional vertigo or positional dizziness $s^{2,3,4}$ provoked by lying down or turning over in the supine position ${ }^{5}$.

B. Duration of attacks $<1 \mathrm{~min}^{6}$.

C. Positional nystagmus ${ }^{7}$ elicited after a brief latency or no latency ${ }^{8}$ by the supine roll test, beating horizontally ${ }^{9}$ toward the undermost ear ${ }^{10,11}$ with the head turned to either side (geotropic direction changing nystagmus) and lasting < $1 \min ^{12,13}$.

D. Not attributable to another disorder ${ }^{14}$.

\section{Notes}

1. Besides attacks of positional vertigo, patients may have prolonged mild unsteadiness.

2. BPPV typically leads to positional vertigo but occasionally patients may complain of positional dizziness.

3. Other complaints during the attacks include external vertigo (the visual sense of environmental motion that often accompanies the internal vestibular sense) [13], unsteadiness, and vegetative symptoms such as nausea, sweating, and tachycardia.

4. Positional vertigo or dizziness must be distinguished from orthostatic symptoms present only on arising but not with other positional triggers [13].

5. Attacks may not only be provoked in bed but also by other head movements (e.g., during rapid lateral head rotations while erect [4], tilting the head backward [i.e., chin upward] or forward). 
6. Patients may overestimate the duration of single attacks and mild, residual symptoms after an attack occasionally last minutes or hours. Furthermore, attacks may be triggered repetitively, causing more extended symptoms. The duration of vertigo should not, however, generally exceed 1 minute; single attacks lasting consistently longer should be considered atypical and spark consideration of alternate or additional diagnoses.

7. If positional nystagmus disappears immediately after positional therapy, this further supports the diagnosis.

8. The latency between the completion of the diagnostic positional maneuver and the onset of positional nystagmus depends on the acceleration of the positional change: the higher the acceleration of the head turn, the shorter the latency and the higher the intensity of nystagmus [4]. With brisk positional maneuvers the latency is typically 1 or 2 seconds. Likewise, the intensity of nystagmus depends on the angle of head rotation: the intensity of nystagmus tends to be higher with larger head rotations in the head roll test [69].

9. The direction of positional nystagmus is predominantly horizontal with a smaller torsional component beating with the upper pole of the eye to the lower ear [2].

10. Nystagmus of lower intensity with reversed direction may appear after the initial positional nystagmus has ceased [4].

11. Probably, canalolithiasis of the horizontal canal may also cause apogeotropic horizontal positional nystagmus. This would be the case when otoconia are located in the anterior part of the horizontal canal (close to the cupula). During the supine roll test to the healthy ear, otoconia would then fall toward or onto the cupula, resulting in transient or long-lasting apogeotropic horizontal positional nystagmus. The supine roll test to the affected ear would provoke transient apogeotropic horizontal positional nystagmus [30 42 62]. In these patients, transformation from apogeotropic to geotropic positional nystagmus can be observed during diagnostic positional maneuvers [21|42 73]. In contrast, persistence of apogeotropic horizontal positional nystagmus following several cycles of the supine roll test is expected with cupulolithiasis.

12. The duration of nystagmus as documented with eye movement recording may exceed 1 minute before it damps spontaneously, however, it does not exceed 2 minutes [2 25].

13. Typically, positional nystagmus increases rapidly in intensity and then declines more slowly (crescendo-decrescendo) [4].

14. History and physical and neurological examinations do not suggest another vestibular disorder or such a disorder is considered but ruled out by appropriate investigations or such disorder is present as a comorbid condition that can be clearly differentiated.

\section{Comments}

Several clinical signs may indicate the side of the affected ear and are confirmatory for the diagnosis. The intensity of nystagmus is usually stronger with the head turned to the affected ear in the supine roll test [4]. Note that the net angle and acceleration of the head rotation should be similar for head turns to the right and left to allow for comparison of nystagmus intensity. Flexing the head forward in the upright position (with the face downward) may elicit a transient nystagmus beating toward the affected ear. Lying backward from the sitting position may provoke transient nystagmus beating toward the healthy ear [23].

Besides positional nystagmus, pseudospontaneous nystagmus (see also comment to 2.3) may also be observed in the upright head position beating either ipsilesional or contralesional [152].

Caloric irrigation of the affected ear [70] and the head thrust test [35] may reveal horizontal canal paresis (possibly due to partial plugging of the canal) that is reversible after successful positional therapy [70].

Transition from geotropic to apogeotropic nystagmus may occur during diagnostic and therapeutic maneuvers, e.g. when the patient bends over from the sitting to the "head-on-knees" position [69]. Transition of canalolithiasis from the posterior canal to the horizontal canal may occur as a result of therapeutic positional maneuvers [36].

\subsection{Cupulolithiasis of the horizontal canal (hc-BPPV-cu)}

A. Recurrent attacks ${ }^{1,2}$ of positional vertigo or positional dizziness ${ }^{3,4,5}$ provoked by lying down or turning over in the supine position. 
B. Positional nystagmus ${ }^{6}$ elicited after a brief latency or no latency by the supine roll test, beating horizontally ${ }^{7}$ toward the uppermost ear with the head turned to either side (apogeotropic direction changing nystagmus), and lasting $>1$ minute $^{8}$.

C. Not attributable to another disorder ${ }^{9}$.

\section{Notes}

1. The duration of an attack of positional vertigo is usually less than 1 minute as patients tend to return the head into a position where vertigo and nystagmus cease. However, the duration can be longer if the head is kept in the provoking position. Furthermore, due to the spatial orientation of the affected cupula, patients may have persisting vertigo or dizziness of lower intensity in the upright position.

2. Besides attacks of positional vertigo, patients may have prolonged mild unsteadiness.

3. BPPV typically leads to positional vertigo but occasionally patients may complain of positional dizziness.

4. Other complaints during the attacks include external vertigo (the visual sense of environmental motion that often accompanies the internal vestibular sense) [13], unsteadiness, and vegetative symptoms such as nausea, sweating, and tachycardia.

5. Positional vertigo or dizziness must be distinguished from orthostatic symptoms present only on arising but not with other positional triggers [13].

6. If positional nystagmus disappears immediately after positional therapy, this further supports the diagnosis.

7. The direction of positional nystagmus is predominantly horizontal with a smaller torsional component beating with the upper pole of the eye to the upper ear [2].

8. Typically, intensity of positional nystagmus builds up slowly over approximately 30 seconds and then gradually decays over a longer period of several minutes [25].

9. History and physical and neurological examinations do not suggest another vestibular disorder or such a disorder is considered but ruled out by appropriate investigations or such disorder is present as a comorbid condition that can be clearly differentiated. As direction-changing apogeotropic positional nystagmus also occurs as a sign of central-vestibular dysfunction, it is mandatory to exclude CNS disease.

\section{Comments}

Several clinical signs can indicate the side of the affected ear and are confirmatory for the diagnosis. The intensity of positional nystagmus is usually stronger with the head turned away from the affected ear in the supine roll test. Note that the net angle and acceleration of the head rotation should be similar for head turns to the right and left to allow for comparison of nystagmus intensity.

Besides positional nystagmus, pseudospontaneous nystagmus may also be observed in the upright head position beating typically to the affected ear [1|12 52]. Pseudospontaneous nystagmus is a form of positional nystagmus that happens to occur with the head in the upright position, making it appear superficially similar to spontaneous nystagmus. In contrast to spontaneous nystagmus, pseudospontaneous nystagmus is strongly influenced by head position and ceases with the head tilted about $30^{\circ}$ forward [12]. Pseudospontaneous nystagmus is attributed to the $30^{\circ}$ angle between the plane of the horizontal canal and the horizontal plane of the head in the upright position that places the ampulla in a higher position than the rest of the canal [12]. Gravity causes deflection of the cupula and produces horizontal nystagmus beating to the side of the horizontal canal affected by cupulolithiasis. Flexing the head $90^{\circ}$ forward in the upright position may elicit pseudospontaneous nystagmus beating toward the healthy ear [12|23]. In the supine position a weak persistent nystagmus beating toward the affected ear may be observed that subsides when the head is turned slightly to that side [12].

Apogeotropic direction changing positional nystagmus is not synonymous with cupulolithiasis but may also occur with canalolithiasis of the horizontal canal [304262 74]. Rapid transition from apogeotropic to geotropic direction changing positional nystagmus during the supine roll test may be due to a variant of canalolithiasis where otoconia are located in the anterior part of the horizontal canal (see canalolithiasis of the horizontal canal). In contrast, persistence of an apogeotropic direction of positional nystagmus with repetitive performance of the supine roll test is supportive of the diagnosis of cupulolithiasis of the horizontal canal. 


\subsection{Probable benign paroxysmal positional vertigo, spontaneously resolved}

A. Recurrent attacks ${ }^{1}$ of positional vertigo or positional dizziness ${ }^{2,3,4}$ provoked by lying down or turning over in the supine position ${ }^{5}$.

B. Duration of attacks $<1 \mathrm{~min}^{6}$.

C. No observable nystagmus and no vertigo with any positional maneuver.

D. Not attributable to another disorder ${ }^{7}$.

\section{Notes}

1. Besides attacks of positional vertigo, patients may have prolonged mild unsteadiness.

2. BPPV typically leads to positional vertigo but occasionally patients may complain of positional dizziness.

3. Other complaints during the attacks include external vertigo (the visual sense of environmental motion that often accompanies the internal vestibular sense) [13], unsteadiness, and vegetative symptoms such as nausea, sweating, and tachycardia.

4. Positional vertigo or dizziness must be distinguished from orthostatic symptoms present only on arising but not with other positional triggers [13].

5. Attacks may not only be provoked in bed but also by other head movements (e.g., tilting the head upward [i.e., chin upward] or forward).

6. Patients may overestimate the duration of single attacks and mild, residual symptoms after an attack occasionally last minutes or hours. Furthermore, attacks may be triggered repetitively, causing more extended symptoms. The duration of vertigo should not, however, generally exceed 1 minute; single attacks lasting consistently longer should be considered atypical and spark consideration of alternate or additional diagnoses.

7. History and physical and neurological examinations do not suggest another vestibular disorder or such a disorder is considered but ruled out by appropriate investigations or such disorder is present as a comorbid condition that can be clearly differentiated.

\section{Comments}

The diagnosis of probable benign paroxysmal positional vertigo that has resolved spontaneously is made during the symptom-free interval. As a consequence, the formerly affected semicircular canal cannot be identified. A history of temporary, isolated episodic positional vertigo with appropriate episode duration and characteristic triggers strongly supports the diagnosis of probable benign paroxysmal positional vertigo that has resolved spontaneously.

The differential diagnosis should include vestibular migraine, which likewise can present with episodic positional vertigo. In contrast to BPPV, episodes of positional vertigo in vestibular migraine tend to be of shorter duration with frequent recurrences, tend to present at an earlier age, and are often accompanied by migraine symptoms (headache, photophobia, phonophobia, migraine aura) [77].

\section{Emerging and controversial syndromes}

The following syndromes are rare variants of BPPV and may be difficult to differentiate from central positional vertigo.

\subsection{Canalolithiasis of the anterior canal ${ }^{1}$ (ac-BPPV)}

A. Recurrent attacks ${ }^{2}$ of positional vertigo or positional dizziness ${ }^{3,4,5}$ provoked by lying down or turning over in the supine position ${ }^{6}$.

B. Duration of attacks $<1 \mathrm{~min}^{7}$.

C. Positional nystagmus ${ }^{8}$ elicited immediately or after a latency of one or few seconds ${ }^{9}$ by the DixHallpike maneuver (on one or both sides) or in the supine straight head-hanging position, beating predominantly vertically downward ${ }^{10}$ and lasting $<1 \mathrm{~min}$.

D. Not attributable to another disorder ${ }^{11}$.

\section{Notes:}

1. The diagnosis of definite canalolithiasis of the anterior canal (3.1.1) can be made on the basis of immediate resolution of positional nystagmus after therapeutic maneuvers. In contrast, probable canalolithiasis of the anterior canal (3.1.2) can be diagnosed only after exclusion of CNS disease when positional nystagmus is refractory to therapeutic maneuvers.

2. Besides attacks of positional vertigo, patients may have prolonged mild unsteadiness. 
3. BPPV typically leads to positional vertigo but occasionally patients may complain of positional dizziness.

4. Other complaints during the attacks include external vertigo (the visual sense of environmental motion that often accompanies the internal vestibular sense) [13], unsteadiness, and vegetative symptoms such as nausea, sweating, and tachycardia.

5. Positional vertigo or dizziness must be distinguished from orthostatic symptoms present only on arising but not with other positional triggers [13].

6. Attacks may not only be provoked in bed but also by other head movements (e.g., tilting the head upward [i.e., chin upward] or forward).

7. Patients may overestimate the duration of single attacks and mild, residual symptoms after an attack occasionally last minutes or hours. Furthermore, attacks may be triggered repetitively, causing more extended symptoms. The duration of vertigo should not, however, generally exceed 1 minute; single attacks lasting consistently longer should be considered atypical and spark consideration of alternate or additional diagnoses.

8. Therapeutic positional maneuvers in canalolithiasis of the anterior canal have been reported as effective [22[81]. Thus, if positional nystagmus disappears immediately after positional therapy, this strongly supports the diagnosis (see note 1). Transition to canalolithiasis of the posterior canal (2.1), canalolithiasis of the horizontal canal (2.2) or cupulolithiasis of the horizontal canal (2.3), either spontaneously or during positional maneuvers, also strongly supports the diagnosis.

9. The latency between the completion of the diagnostic positional maneuver and the onset of positional nystagmus may be as long as 30 seconds in rare cases [20 57].

10. The positional nystagmus may have a small torsional component, beating with the upper pole of the eye toward the affected ear [2].

11. History and physical and neurological examinations do not suggest another vestibular disorder or such a disorder is considered but ruled out by appropriate investigations or such disorder is present as a comorbid condition that can be clearly differentiated. As positional downbeating nystagmus also occurs as a sign of central vestibular dysfunction, it is mandatory to exclude CNS disease if positional nystagmus does not cease promptly after therapeutic maneuvers.

\section{Comments}

Canalolithiasis of the anterior canal is rare compared to posterior and horizontal canal variants [3860 81]. This is probably related to the anatomical orientation of the anterior canal, which allows particles to leave the canal simply after lying down and sitting up again.

Positional nystagmus can be provoked in the supine position with the head hanging straight below the earth-horizontal and by Dix-Hallpike positioning, that, no matter to which side the head is turned, stimulates particle migration within the affected anterior canal [2616]. In the Dix-Hallpike position, nystagmus may be stronger or exclusively present with the affected ear up or down. The most sensitive diagnostic test for BPPV of the anterior canal seems to be the straight head-hanging position [20|22]. Thus, in canalolithiasis of the anterior canal, the direction of the torsional component, rather than the side of the Dix-Hallpike maneuver, indicates the affected side. However, the small torsional component of the positional nystagmus can be easily missed in clinical practice, rendering identification of the affected side unreliable [26]. Identification of the torsional component of nystagmus may be facilitated by eye movement recording with video-oculography [16/22] or search-coils [2].

Attenuation of positional nystagmus with repeated positional maneuvers (fatigability) may be supportive of the diagnosis of canalolithiasis of the anterior canal, but it is not present in all patients [20 57].

Transient downbeating positional nystagmus can also occasionally be observed after treatment of BPPV of the posterior canal, when the Dix-Hallpike maneuver is performed. This finding may be due to translocation of otoconia into the anterior canal or to otoconia that have not left the posterior canal completely during therapeutic positional maneuvers, dropping back into the canal during the Dix-Hallpike maneuver, and thus inducing an inhibitory nystagmus that is downbeating [75].

\subsection{Cupulolithiasis of the posterior canal (pc-BPPV-cu)}

A. Recurrent attacks ${ }^{1,2}$ of positional vertigo or positional dizziness ${ }^{3,4,5}$ provoked by lying down or turning over in the supine position ${ }^{6}$.

B. Positional nystagmus ${ }^{7}$ elicited after a brief or no latency by a "half Dix-Hallpike maneuver", beating torsionally with the upper pole of the eye to the lower ear and vertically upward (to the forehead) and lasting $>1 \mathrm{~min}$.

C. Not attributable to another disorder ${ }^{9}$. 


\section{Notes:}

1. The duration of an attack of positional vertigo is usually less than 1 minute as patients tend to return the head into a position where vertigo and nystagmus cease. However, the duration can be longer when the head is kept in the provoking position.

2. Besides attacks of positional vertigo, patients may have prolonged mild unsteadiness.

3. BPPV typically leads to positional vertigo but occasionally patients may complain of positional dizziness.

4. Other complaints during the attacks include external vertigo (the visual sense of environmental motion that often accompanies the internal vestibular sense) [13], unsteadiness, and vegetative symptoms such as nausea, sweating, and tachycardia.

5. Positional vertigo or dizziness must be distinguished from orthostatic symptoms present only on arising but not with other positional triggers [13].

6. Attacks may not only be provoked in bed but also by other head movements (e.g., tilting the head upward [i.e., chin upward] or forward).

7. If positional nystagmus disappears immediately after positional therapy, this further supports the diagnosis.

8. A "half Dix-Hallpike maneuver" is performed with the head turned $45^{\circ}$ toward the side to be tested and resting slightly raised from supine (about $30^{\circ}$ in flexion). This position is best suited to bring the affected cupula to an earth-horizontal position to be maximally deflected by the gravitational force [29].

9. History and physical and neurological examinations do not suggest another vestibular disorder or such a disorder is considered but ruled out by appropriate investigations or such disorder is present as a comorbid condition that can be clearly differentiated.

\section{Comments}

Cupulolithiasis of the posterior canal has rarely been described [43]. Positional nystagmus may disappear when the head is further reclined in the Dix-Hallpike position [43]. A "reversed Dix-Hallpike maneuver" with the head bent forward $90^{\circ}$ from the upright posi- tion after being turned $45^{\circ}$ to the affected side may reveal nystagmus beating in the opposite direction compared to the "half Dix-Hallpike maneuver" [29].

\subsection{Lithiasis of multiple canals (mc-BPPV)}

A. Recurrent attacks ${ }^{1}$ of positional vertigo or positional dizziness ${ }^{2,3,4}$ provoked by lying down or turning over in the supine position ${ }^{5}$.

B. Duration of attacks $<1 \mathrm{~min}^{6,7}$.

C. Positional nystagmus ${ }^{8,9}$ compatible with canalolithiasis of more than one canal during the DixHallpike maneuver and the supine roll test.

D. Not attributable to another disorder ${ }^{10}$.

\section{Notes:}

1. Besides attacks of positional vertigo, patients may have prolonged mild unsteadiness.

2. BPPV typically leads to positional vertigo but occasionally patients may complain of positional dizziness.

3. Other complaints during the attacks include external vertigo (the visual sense of environmental motion that often accompanies the internal vestibular sense) [13], unsteadiness, and vegetative symptoms such as nausea, sweating, and tachycardia.

4. Positional vertigo or dizziness must be distinguished from orthostatic symptoms present only on arising but not with other positional triggers [13].

5. Attacks may not only be provoked in bed but also by other head movements (e.g., tilting the head upward [i.e., chin upward] or forward).

6. This criterion is not required when the labyrinth is affected by cupulolithiasis.

7. Patients may overestimate the duration of single attacks and mild, residual symptoms after an attack occasionally last minutes or hours. Furthermore, attacks may be triggered repetitively, causing more extended symptoms. The duration of vertigo should not, however, generally exceed 1 minute; single attacks lasting consistently longer should be considered atypical and spark consideration of alternate or additional diagnoses.

8. If positional nystagmus disappears immediately after positional therapy, this further supports the diagnosis. 
9. The most frequent combination is a canalolithiasis of the posterior and horizontal canal of one labyrinth. This may result in a transient positional nystagmus with horizontal and torsional components of equal intensity in the DixHallpike maneuver [2]. Alternatively, a mixed torsional-vertical positional nystagmus compatible with excitation of the posterior canal in the Dix-Hallpike maneuver on one side and a predominantly horizontal positional nystagmus in the supine roll test to both sides may be observed in the same session [8]. Other combinations of lithiasis of multiple canals may also occur but are rarer.

10. History and physical and neurological examinations do not suggest another vestibular disorder or such a disorder is considered but ruled out by appropriate investigations or such disorder is present as a comorbid condition that can be clearly differentiated.

\section{Comments}

Lithiasis of multiple canals is probably common, affecting up to $20 \%$ of patients with BPPV [56 60]. Lithiasis of multiple canals probably occurs most often after head trauma [8].

\subsection{Possible benign paroxysmal positional vertigo}

A. Attacks of positional vertigo missing one of the criteria of a disorder coded above.

B. Not attributable to another disorder ${ }^{1}$.

\section{Notes:}

1. History and physical and neurological examinations do not suggest another vestibular disorder or such a disorder is considered but ruled out by appropriate investigations or such disorder is present as a comorbid condition that can be clearly differentiated.

\section{Comments}

This category may include e.g. (i) patients with positional vertigo without observable positional nystagmus despite eye movement recording or with atypical po- sitional nystagmus that nonetheless ceases after therapeutic positioning, (ii) patients with presumed involvement of multiple semicircular canals that cannot be specified, (iii) patients with simultaneous occurrence of peripheral and central positional nystagmus [7].

Do not code as 3.4. possible benign paroxysmal positional vertigo if the patient fulfills the criteria for 1.4. probable benign paroxysmal positional vertigo, spontaneously resolved.

\section{Acknowledgements}

This work was supported by travelling grants from the Bárány Society and from Neuro+ Berlin, a nonprofit association for neurological research. We thank the Japan Society of Equilibrium Research, the American Neurotology Society and the members of the Bárány Society for valuable suggestions. Michael von Brevern wishes to thank Alexandre Bisdorff and Thomas Lempert for many fruitful discussions on the classification of BPPV.

\section{References}

[1] G. Asprella-Libonati, Pseudo-spontaneous nystagmus: A new sign to diagnose the affected side in lateral semicircular canal benign paroxysmal positional vertigo, Acta Otorhinolaryngol Ital 28 (2008), 73-78.

[2] S.T. Aw, M.J. Todd, G.E. Aw, L.A. McGarvie and G.M. Halmagyi, Benign paroxysmal nystagmus. A study of its three-dimensional spatio-temporal characteristics, Neurology 64 (2005), 1897-1905.

[3] R.W. Baloh, V. Honrubia and K. Jacobson, Benign positional vertigo: Clinical and oculographic features in 240 cases, $\mathrm{Neu}$ rology 37 (1987), 371-378.

[4] R.W. Baloh, K.M. Jacobson and V. Honrubia, Horizontal semicircular canal variant of benign positional vertigo, $\mathrm{Neu}$ rology 43 (1993), 2542-2549.

[5] R.W. Baloh, Q. Yue, K.M. Jacobson and V. Honrubia, Persistent direction-changing positional nystagmus: Another variant of benign paroxysmal positional vertigo? Neurology 45 (1995), 1297-1301.

[6] P. Bertholon, A.M. Bronstein, R.A. Davies, P. Rudge and K.V. Thilo, Positional down beating nystagmus in 50 patients: Cerebellar disorders and possible anterior canal semicircular canalolithiasis, J Neurol Neurosurg Psychiatry 72 (2002), 366-372.

[7] P. Bertholon, J.C. Antoine, C. Martin and D. Michel, Simultaneous occurrence of a central and a peripheral positional nystagmus during the Dix-Hallpike manœuvre, Eur Neurol 50 (2003), 248-250.

[8] P. Bertholon, L. Chelikh, S. Tringali, A.P. Timoshenko and C. Martin, Combined horizontal and posterior canal benign paroxysmal positional vertigo in three patients with head trauma, Ann Otol Rhinol Laryngol 114 (2005), 105-110. 
[9] P. Bertholon, S. Tringali, M.B. Faye, J.C. Antoine and C. Martin, Prospective study of positional nystagmus in 100 consecutive patients. Ann Otol Rhinol Laryngol 115 (2006), 587-594.

[10] J.A. Beyea, S.K. Agrawal and L.S. Parnes, Transmastoid semicircular canal occlusion: A safe and highly effective treatment for benign paroxysmal positional vertigo and superior canal dehiscence. Laryngoscope 122 (2012), 1862-1866.

[11] N. Bhattacharyya, R.F. Baugh, L. Orvidas, D. Barrs, L.J. Bronstein, S. Cass, A.A. Chalian, A.L. Desmond, J.M. Earll, T.D. Fife, D.J. Fuller, J.O. Judge, N.R. Mann, R.M. Rosenfeld, L.T. Schuring, R.W. Steiner, S.L. Whitney and J. Haidari, Clinical practice guideline: Benign paroxysmal positional vertigo, Otolaryngol Head Neck Surg 139 (2008), S47-S81.

[12] A. Bisdorff and D. Debatisse, Localizing signs in positional vertigo due to lateral canal cupulolithiasis, Neurology 57 (2001), 1085-1088.

[13] A. Bisdorff, M. von Brevern, T. Lempert and D.E. NewmanToker, Classification of vestibular symptoms: towards an international classification of vestibular disorders, J Vest Res 19 (2009), 1-13.

[14] T. Brandt, Positional and positioning vertigo and nystagmus, J Neurol Sci 95 (1990), 3-28.

[15] T. Brandt and S. Steddin, Current view of the mechanism of benign paroxysmal positional vertigo: Cupulolithiasis or canalolithiasis? J Vest Res 3 (1993), 373-382.

[16] K. Brantberg and J. Bergenius, Treatment of anterior canal benign paroxysmal positional vertigo by canal plugging: A case report, Acta Otolaryngol 122 (2002), 28-30.

[17] R.A. Buckingham, Anatomical and theoretical observations on otolith repositioning for benign paroxysmal positional vertigo, Laryngoscope 109 (1999), 717-722.

[18] B. Bürki, L. Simon, S. Garab, Y. Lundberg, H. Jünger and D. Straumann, Sitting-up vertigo and trunk retropulsion in patients with benign positional vertigo but without positional nystagmus, J Neurol Neurosurg Psychiatry 82 (2011), 98104.

[19] U. Büttner, C. Helmchen and T. Brandt, Diagnostic criteria for central versus peripheral positioning nystagmus: A review, Acta Otolaryngol 119 (1999), 1-5.

[20] J. Cambi, S. Astore, M. Mandalà, F. Trabalzini and D. Nuti, Natural course of positional down-beating nystagmus of peripheral origin, J Neurol 260 (2013), 1489-1496.

[21] A.P. Casani, G. Vannucci, B. Fattori and S. Berrettini, The treatment of horizontal canal positional vertigo: Our experience in 66 cases, Laryngoscope 112 (2002), 172-178.

[22] A.P. Casani, N. Cerchiai, I. Dallan and S. Sellari-Franceschini, Anterior canal lithiasis: Diagnosis and treatment, Otolaryngol Head Neck Surg 144 (2011), 412-418.

[23] Y.H. Choung, Y.R. Shin, H. Kahng, K. Park and S.J. Choi, "Bow and lean test" to determine the affected ear of horizontal canal benign paroxysmal positional vertigo, Laryngoscope 116 (2006), 1776-1781.

[24] H.S. Cohen, Side-lying as an alternative to the Dix-Hallpike test of the posterior canal, Otol Neurotol 25 (2004), 130-134.

[25] G. de la Meilleure, I. Dehaene, M. Depondt, W. Damman, L. Crevits and G. Vanhooren, Benign paroxysmal positional vertigo of the horizontal canal, J Neurol Neurosurg Psychiatry 60 (1996), 68-71.

[26] S. di Girolamo, G. Paludetti, G. Briglia, A. Cosenca, R. Santarelli and W. Di Nardo, Postural control in benign paroxysmal positional vertigo before and after recovery, Acta Otolaryngol 118 (1998), 289-293.

[27] M.R. Dix and C.S. Hallpike, The pathology, symptomatology and diagnosis of certain common disorders of the vestibular system, Proc R Soc Med 45 (1952), 341-354.

[28] J. Epley, Positional vertigo related to semicircular canalolithiasis, Otolaryngol Head Neck Surg 112 (1995), 154-161.

[29] J.M. Epley, Human experience with canalith repositioning maneuvers, Ann N Y Acad Sci 942 (2001), 179-191.

[30] T. Fife, Recognition and management of horizontal canal benign positional vertigo, Am J Otol 19 (1998), 345-351.

[31] T.D. Fife, D.J. Iverson, T. Lempert, J.M. Furman, R.W. Baloh, R.J. Tusa, S. Herdman, M.J. Morrow and G.S. Gronseth, Therapies for benign paroxysmal positional vertigo (an evidence-based review). Report of the Quality Standards Subcommittee of the American Academy of Neurology, Neurology 70 (2008), 2067-2074.

[32] J.M. Furman, S.P. Cass and B.C. Briggs, Treatment of benign positional vertigo using heels-over-head rotation, Ann Otol Rhinol Laryngol 107 (1998), 1046-1053.

[33] T.C. Hain, T.M. Squires and H.A. Stone, Clinical implications of a mathematical model of benign paroxysmal positional vertigo, Ann N Y Acad Sci 1039 (2005), 384-394.

[34] D.S. Haynes, J.R. Resser, R.F. Labadie, C.R. Girasole, B.T. Kovach, L.E. Scheker and D.C. Walker, Treatment of benign paroxysmal positional vertigo using the Semont maneuver: Efficacy in patients presenting without nystagmus, Laryngoscope 112 (2002), 796-801.

[35] K.D. Heidenreich, K. Beaudoin and J.A. White, Can active lateral canal benign paroxysmal positional vertigo mimic a false-positive head thrust test? Am J Otolaryngol 30 (2009), 353-355.

[36] S.J. Herdmann and R.J. Tusa, Complications of the canalith repositioning procedure, Arch Otolaryngol Head Neck Surg 122 (1996), 281-286.

[37] K. Hiruma, T. Numata, T. Mituhashi, T. Tomemori, R. Watanabe and Y. Okamoto, Two types of direction-changing positional nystagmus with neutral points, Auris Nasus Larynx 38 (2011), 46-51.

[38] V. Honrubia, R.W. Baloh, M.R. Harris and K.M. Jacobson, Paroxysmal positional vertigo syndrome, Am J Otol 20 (1999), 465-470.

[39] V. Honrubia and M. House, Mechanism of posterior semicircular canal stimulation in patients with benign paroxysmal positional vertigo, Acta Otolaryngol 121 (2001), 234-40.

[40] M.G. House and V. Honrubia, Theoretical models for the mechanisms of benign paroxysmal positional vertigo, Audiol Neurootol 8 (2003), 91-99.

[41] T. Imai, M. Ito, N. Takeda, A. Uno, T. Matsunaga, K. Sekine and T. Kubo, Natural course of the remission of vertigo in patients with benign paroxysmal positional vertigo, Neurology 64 (2005), 920-921.

[42] T. Imai, N. Takeda, G. Sato, K. Sekine, M. Ito, K. Nakamae and T. Kubo, Changes in slow phase eye velocity and time constant of positional nystagmus at transformation from cupulolithiasis to canalolithiasis, Acta Otolaryngol 128 (2008), 22-28.

[43] T. Imai, N. Takeda, M. Ito, K. Sekine, G. Sato, Y. Midoh, K. Nakamae and T. Kubo, 3D analysis of benign positional nystagmus due to cupulolithiasis in posterior semicircular canal, Acta Otolaryngol 129 (2009), 1044-1049.

[44] T. Imai, N. Takeda and A. Uno, Benign paroxysmal positional vertigo showing sequential translations of four types of nystagmus, Auris Nasus Larynx 39 (2012), 544-548.

[45] L.E. Jackson, B. Morgan, J.R. Fletcher and W.W. Krueger, Anterior canal benign paroxysmal positional vertigo: an underappreciated entity, Otol Neurotol 28 (2007), 218-222. 
[46] K. Johkura, Central paroxysmal positional vertigo: Isolated dizziness caused by small cerebellar hemorrhage, Stroke $\mathbf{3 8}$ (2007), e26-e27.

[47] A. Katsarkas, Benign paroxysmal positional vertigo (BPPV): Idiopathic versus post-traumatic, Acta Otolaryngol 119 (1999), 745-749.

[48] H.A. Kim, H.A. Yi and H. Lee, Apogeotropic central positional nystagmus as a sole sign of nodular infarction, Neurol Sci 33 (2012), 1189-1191.

[49] J.F. Kveton and M. Kashgarian, Particulate matter within the membranous labyrinth: Pathologic or normal? Am J Otol 15 (1994), 173-176.

[50] D.J. Lanska and B. Remler, Benign paroxysmal positional vertigo. Classic descriptions, origins of the provocative positioning technique, and conceptual develepments, Neurology 48 (1997), 1167-1177.

[51] C. Lechner, R.L. Taylor and C. Todd, Causes and characteristics of horizontal positional nystagmus, J Neurol 261 (2014), 1009-1017.

[52] S.U. Lee, H.J. Kim and J.S. Kim, Pseudo-spontaneous and head-shaking nystagmus in horizontal canal benign paroxysmal positional vertigo, Otol Neurotol 35 (2014), 495-500.

[53] T. Lempert and K. Tiel-Wilck, A positional manoeuvre for treatment of horizontal-canal benign positional vertigo, Laryngoscope 106 (1996), 476-478.

[54] T. Lempert, C. Wolsley, R. Davies, M.A. Gresty and A.M. Bronstein, Three hundred sixty-degree rotation of the posterior semicircular canal for treatment of benign positional vertigo: A placebo-controlled trial, Neurology 49 (1997), 729 733.

[55] J.A. Lopez-Escamez, M.J. Gamiz, A. Fernandez-Perez, M. Gomez-Finana and I. Sanchez-Canet, Impact of treatment on healthrelated quality of life in patients with posterior canal benign paroxysmal positional vertigo, Otol Neurotol 24 (2003), 637-641.

[56] J.A. Lopez-Escamez, M.I. Molina, M.J. Gamiz, A.J. Fernandez-Perez, M. Gomez, M.J. Palma and C. Zapata, Multiple positional nystagmus suggests multiple canal involvement in benign paroxysmal positional vertigo, Acta Otolaryngol 125 (2005), 954-961.

[57] J.A. Lopez-Escamez, M.I. Molina and M.J. Gamiz, Anterior semicircular canal benign paroxysmal positional vertigo and positional downbeating nystagmus, Am J Otolaryngol 27 (2006), 173-178

[58] L. Luis, J. Costa, F. Vaz Garcia, J. Valls-Solé, T. Brandt and E. Schneider, Spontaneous plugging of the horizontal semicircular canal with reversible canal dysfunction and recovery of vestibular evoked myogenic potentials, Otol Neurotol 34 (2013), 743-747.

[59] B. Moriarty, J. Rutka and M. Hawke, The incidence and distribution of cupulae deposits in the labyrinth. Laryngoscope 102 (1992), 56-59.

[60] M. Nakayama and J. Epley, BPPV and variants: Improved treatment results with automated, nystagmus-based repositioning, Otolaryngol Head Neck Surg 133 (2005), 107-112.

[61] R.A. Nunez, S.P. Cass and J.M. Furman, Short and long-term outcomes of canalith repositioning for benign paroxysmal positional vertigo, Otolaryngology Head Neck Surg 122 (2000), 647-652.

[62] D. Nuti, P. Vannucchi and P. Pagnini, Benign paroxysmal positional vertigo of the horizontal canal: A form of canalolithiasis with variable clinical features, J Vest Res 6 (1996), 173184
63] K. Otsuka, M. Suzuki and M. Furaya, Model experiment of benign paroxysmal positional vertigo mechanism using the whole membraneous labyrinth, Acta Otolaryngol 123 (2003), 515-518.

[64] L.S. Parnes and J.A. McClure, Free-floating endolymphatic particles: A new operative finding during posterior semicircular canal occlusion. Laryngoscope 102 (1992), 988-992.

[65] S.M. Rajguru, M.A. Ifediba and R.D. Rabbitt, Three-dimensional biomechanical model of benign paroxysmal positional vertigo, Ann Biomed Eng 32 (2004), 831-846.

[66] M. Ruckenstein, Therapeutic efficacy of the Epley canalith repositioning maneuver, Laryngoscopy 111 (2001), 940-945.

[67] H.F. Schuknecht, Cupulolithiasis. Arch Otolaryngol 90 (1969), 765-778.

[68] S. Steddin and T. Brandt, Unilateral mimicking bilateral benign paroxysmal positioning vertigo, Arch Otolaryngol Head Neck Surg 120 (1994), 1339-1341.

[69] S. Steddin and T. Brandt, Horizontal canal benign paroxysmal positioning vertigo (h-BPPV): Transition from canalolithiasis to cupulolithiasis, Ann Neurol 40 (1996), 918-922.

70] M. Strupp, T. Brandt and S. Steddin, Horizontal canal benign paroxysmal positioning vertigo: Reversible ipsilateral caloric hypoexcitability caused by canalolithiasis? Neurology 45 (1995), 2072-2076

[71] M. Suzuki, A. Kadir, N. Hayashi and M. Takamoto, Functional model of benign paroxysmal positional vertigo using an isolated frog semicircular canal, J Vest Res 6 (1996), 121-125.

72] G. Tirelli, E. d'Orlando, V. Giacomarra and M. Russolo, Benign positional vertigo without detectable nystagmus, Laryngoscope 111 (2001), 1053-1056.

[73] P. Vannucchi and R. Pecci, Pathophysiology of lateral semicircular canal paroxysmal positional vertigo, $J$ Vest Res 20 (2010), 433-438.

[74] P. Vannucchi and R. Pecci, About nystagmus transformation in a case of apogeotropic lateral semicircular canal benign paroxysmal positional vertigo. Int J Otolaryngol (2011), 2011: 687921. doi: 10.1155/2011/687921.

[75] P. Vannucchi, R. Pecci and B. Giannoni, Posterior semicircular canal benign paroxysmal positional vertigo presenting with torsional downbeating nystagmus: An apogeotropic variant, Int J Otolaryngol; 2012: 413603. doi: $10.1155 / 2012 / 413603$.

[76] M. von Brevern, A. Clarke and T. Lempert, Continous vertigo and spontaneous nystagmus due to canalolithiasis of the horizontal canal, Neurology 56 (2001), 684-686.

[77] M. von Brevern, A. Radtke, A. Clarke and T. Lempert, Migrainous vertigo presenting with episodic positional vertigo, Neurology 62 (2004), 469-472.

[78] M. von Brevern, T. Seelig, H. Neuhauser and T. Lempert, Benign paroxysmal positional vertigo predominantely affects the right labyrinth, J Neurol Neurosurg Psychiatry 75 (2004), 1487-1488.

[79] M. von Brevern, A. Radtke, F. Lezius, M. Feldmann, T. Ziese, T. Lempert and H. Neuhauser, Epidemiology of benign paroxysmal positional vertigo: A population based study, J Neurol Neursurg Psychiatry 78 (2007), 710-715.

[80] D.B. Welling, L.S. Parnes, B. O’Brien, L.O. Bakaletz, D.E. Brackmann and R. Hinojosa, Particulate matter in the posterior semicircular canal. Laryngoscope 107 (1997), 90-94.

[81] D.A. Yacovino, T.C. Hain and F. Gualtieri, New therapeutic maneuver for anterior canal benign paroxysmal positional vertigo, J Neurol 256 (2009), 1851-1855. 